\title{
Technical aspects of the evaluation of the overlap of Hartree-Fock-Bogoliubov wave functions
}

\author{
L. M. Robledo* \\ Departamento Física Teórica, Universidad Autónoma de Madrid, E-28049 Madrid, Spain
}

(Received 10 February 2011; published 8 July 2011)

\begin{abstract}
Several technical aspects concerning the evaluation of the overlap between two mean-field wave functions of the Hartree-Fock-Bogoliubov type are discussed. The limit when several orbitals become fully occupied is derived as well as the formula to reduce the dimensionality of the problem when exactly empty orbitals are present. The formalism is also extended to deal with different bases for each of the wave functions. Several practical results concerning the evaluation of pfaffians, as well as the canonical decomposition of norm overlaps, are also discussed in the Appendices.
\end{abstract}

DOI: 10.1103/PhysRevC.84.014307

PACS number(s): 21.60.Jz

\section{INTRODUCTION}

To compute the modulus and phase of the overlap of two mean field wave functions of the Hartree-Fock-Bogoliubov (HFB) type, a new formulation, based on the pfaffian of a skew-symmetric matrix, was recently proposed [1]. It improves on previous treatments of the problem as described in Refs. [2,3]. The new formulation is based on the powerful concept of fermion coherent states [4-8] and involves the evaluation of a quantity called the pfaffian of a skew-symmetric matrix - see, for instance [9] for a definition of the pfaffian in a physical context - that is similar in spirit (linear combination of products of matrix elements) to the determinant of a general matrix. In the derivation [1] of the formula for the overlap it is assumed that the two mean-field wave functions of the HFB type (see [10] for definition and properties) can be related to a common reference one (usually chosen as the true particle vacuum) by means of their Thouless parametrization. It is very likely to find cases where the Thouless parametrization is ill defined because, for that particular case, it involves the inverse of a (near) singular matrix. This situation corresponds to the presence of particles (or quasiparticles in the general case of an arbitrary HFB vacuum) that have an occupancy of one, rendering the wave function orthogonal to the common (or reference) wave function. To handle those singular cases it was suggested in [1] to just change the common reference mean field wave function in order to modify the occupancies with the hope that none of them will be close to one. However, it is desirable to have an alternative for those cases where the change of reference wave function is either not possible or too cumbersome to carry out. Therefore, the formal limit of occupancies going to one is considered and a formula which is well defined in that limit is derived. Using the same kind of ideas, the case where some particles (or quasiparticles) have zero occupancy is also handled. Those excitations do not contribute to the overlap and therefore the formula for the overlap involves matrices smaller than the original ones. The new formula should be regarded as a thrifty alternative to the original formula for those situations where the wave functions are expanded in huge bases. Similar manipulations

\footnotetext{
*luis.robledo@uam.es
}

to reduce the size of matrices and/or deal with full occupancies, were considered in Refs. [11-14]. In addition to the mentioned limits, the case where each of the mean field wave functions are expressed in different single-particle basis is considered. The two bases are related to each other by a general (not necessarily unitary) transformation. The result obtained is useful, for instance, to compute the overlap of the operators for spatial transformations (as translations or rotations) between arbitrary mean field HFB wave functions. The result is general enough as to allow for transformations that do not map the single particle basis into itself (noncomplete basis under the transformation). This was already considered in [11] in a general framework. However, in [11] the arbitrariness in the phase of the overlap was not considered at all. In [14] the implications of considering two different bases was also addressed, but there it was implicitly assumed that both bases shared the same block structure defining the conjugate states. This is a rather stringent assumption that does not hold in general as, for instance, in the case of time reversal breaking cranking wave functions. Finally, some useful results concerning the formal evaluation of pfaffians are discussed in the appendices. These results can be of interest elsewhere as the use of pfaffians is becoming increasingly popular [15-17]. To cover also more practical aspects, the reader is referred to [18] for a thorough description of useful algorithms to compute numerically and symbolically the pfaffian of arbitrary skew symmetric matrices.

The relevance of the present formulation is a direct consequence of the increasing popularity of the so-called "beyond mean-field methods" in nuclear physics [12,19-23] that demand the evaluation of both the modulus and phase of the overlaps between arbitrary HFB wave functions. A reliable determination of the sign of the norm can also be useful in to order to pin down the location of the zeros of the HFB overlaps [24]. The location of the zeros (nodal lines) can be a useful piece of information in assessing the impact of the so-called "pole problem" that plagues present beyond mean-field calculations [25-27] and could help to envisage strategies to avoid it.

In Sec. II the formulas pertaining the two limits considered are derived and their implications discussed. The procedure to change to a different common reference HFB vacuum that could be an easy alternative, in some cases, is also 
discussed. In Sec. III the case where the two HFB wave functions are expressed in different bases is discussed. Finally, in Appendices A, B, and C some relevant results required in the derivations are discussed.

\section{EVALUATION OF THE OVERLAP IN VARIOUS LIMITS}

To begin with, it is convenient to remind the reader about the notation used and the results obtained in [1]. The goal is to evaluate both the modulus and phase of the overlap $\left\langle\phi_{0} \mid \phi_{1}\right\rangle$ between two HFB wave functions $\left|\phi_{0}\right\rangle$ and $\left|\phi_{1}\right\rangle$ which are given in terms of the Thouless parametrization of a general HFB wave function

$$
\left|\phi_{i}\right\rangle=\exp \left(\frac{1}{2} \sum_{k k^{\prime}} M_{k k^{\prime}}^{(i)} a_{k}^{+} a_{k^{\prime}}^{+}\right)|0\rangle .
$$

The skew-symmetric matrices $M^{(i)}=\left(V^{(i)} U^{(i)-1}\right)^{*}$, of dimension $N \times N$ ( $N$ is assumed to be an even number $N=2 q$ as required for fermions), are built from to the coefficients $U^{(i)}$ and $V^{(i)}$ of the Bogoliubov transformation defining the quasiparticle annihilation operators

$$
\alpha_{k}^{(i)}=\sum_{l} U_{l k}^{(i) *} a_{l}+V_{l k}^{(i) *} a_{l}^{+}
$$

associated to $\left|\phi_{i}\right\rangle$. The above wave functions are normalized to have $\left\langle 0 \mid \phi_{i}\right\rangle=1$ instead of the more traditional normalization. As shown in [1] the overlap can be written as

$$
\left\langle\phi_{0} \mid \phi_{1}\right\rangle=S_{N} \mathrm{pfM}
$$

in terms of the phase $S_{N}=(-1)^{N(N+1) / 2}$ and the $2 N \times 2 N$ skew-symmetric matrix

$$
\mathbb{M}=\left(\begin{array}{cc}
M^{(1)} & -\mathbb{I} \\
\mathbb{I} & -M^{(0) *}
\end{array}\right) .
$$

To obtain the results of the present section the Bloch-Messiah decomposition of the Bogoliubov amplitudes [10], namely $U^{(i)}=D^{(i)} \bar{U}^{(i)} C^{(i)}$ and $V^{(i)}=D^{(i) *} \bar{V}^{(i)} C^{(i)}$, is used. In the previous expressions, $D^{(i)}$ and $C^{(i)}$ are given unitary matrices and $\bar{U}^{(i)}$ and $\bar{V}^{(i)}$ are real matrices with special diagonal forms. By using this decomposition we can write

$$
M^{(i)}=D^{(i)} M_{C}^{(i)} D^{(i) T},
$$

where the skew-symmetric matrix $M_{C}^{(i)}$ is in "skew-symmetric diagonal" (or canonical) form

$$
M_{C}^{(i)}=\left(\begin{array}{cc}
0 & \bar{M}^{(i)} \\
-\bar{M}^{(i)} & 0
\end{array}\right) .
$$

The connection between the diagonal matrix $\bar{M}^{(i)}$ and the $\bar{U}^{(i)}$ and $\bar{V}^{(i)}$ ones is evident in its matrix elements

$$
\bar{M}_{j k}^{(i)}=\frac{v_{j}^{(i)}}{u_{j}^{(i)}} \delta_{j k} .
$$

The extreme values of the ratios $v_{j}^{(i)} / u_{j}^{(i)}$ are infinity for fully occupied levels $\left(v^{(i)}=1\right)$ or zero for empty levels $\left(v^{(i)}=0\right)$.
For further developments it is convenient to single out those values and write

$$
\bar{M}^{(i)}=\left(\begin{array}{cc}
\bar{N}^{(i)} & 0 \\
0 & \bar{O}^{(i)}
\end{array}\right),
$$

where the diagonal matrix $\bar{O}^{(i)}$ contains the $K^{(i)}$ diagonal elements belonging to the extreme values, infinity or zero, mentioned above. The dimension of this matrix is $K^{(i)} \times K^{(i)}$ and depends on the kind of extreme value considered. Each of the two limiting cases require different considerations and hence we will from now on considered them separately.

\section{A. Limit of fully occupied levels}

In this case, there are $K^{(i)}$ fully occupied levels in each of the HFB wave functions $\left|\phi_{i}\right\rangle$ and the corresponding diagonal elements of the matrices $\bar{M}^{(i)}$ [the ones corresponding to $\bar{O}^{(i)}$ in Eq. (8)] tend to infinity. This is a serious challenge, as the overlap of Eq. (3) and the norm of the $\left|\phi_{i}\right\rangle$ diverge. The divergence has to be regularized and singled out of the overlap in order to cancel it out with the diverging factors coming from the norms of the HFB wave functions. To this end we write $M_{C}^{(i)}=R^{(i)} M_{C R}^{(i)} R^{(i) T}$ where we have introduced the "canonical regularized" (CR) matrix

$$
M_{C R}^{(i)}=\left(\begin{array}{cc|cc} 
& & \bar{N}^{(i)} & 0 \\
& & 0 & \mathbb{I}_{K^{(i)}} \\
\hline-\bar{N}^{(i)} & 0 & & \\
0 & -\mathbb{I}_{K^{(i)}} & &
\end{array}\right)
$$

as well as

$$
R^{(i)}=\left(\begin{array}{cccc}
\mathbb{I}_{N-K^{(i)}} & 0 & 0 & 0 \\
0 & \mathbb{I}_{K^{(i)}} & 0 & 0 \\
0 & 0 & \mathbb{I}_{N-K^{(i)}} & 0 \\
0 & 0 & 0 & \bar{O}^{(i)}
\end{array}\right) .
$$

In all the cases $\mathbb{I}_{K^{(i)}}$ represents the unit matrix of dimension $K^{(i)}$. With the above definitions the matrix $\mathbb{M}$ of Eq. (4), which enters the expression of Eq. (3) for the overlap, can be factorized as

$$
\mathbb{M}=\left(\begin{array}{cc}
\tilde{R}^{(1)} & 0 \\
0 & \tilde{R}^{(0) *}
\end{array}\right)\left(\begin{array}{cc}
M_{C R}^{(1)} & -S \\
S^{T} & -M_{C R}^{(0) *}
\end{array}\right)\left(\begin{array}{cc}
\tilde{R}^{(1) T} & 0 \\
0 & \tilde{R}^{(0)+}
\end{array}\right),
$$

where

$$
\tilde{R}^{(i)}=D^{(i)} R^{(i)}
$$

and

$$
S=\tilde{R}^{(1)-1}\left(\tilde{R}^{(0)+}\right)^{-1} .
$$

Using now the property $\operatorname{pf}\left(B^{T} A B\right)=\operatorname{det}(B) \operatorname{pf}(A)$ we obtain

$$
\operatorname{pf}(\mathbb{M})=\operatorname{det}\left(\tilde{R}^{(1)}\right) \operatorname{det}\left(\tilde{R}^{(0) *}\right) \operatorname{pf}(\tilde{\mathbb{M}})
$$

with

$$
\tilde{\mathbb{M}}=\left(\begin{array}{cc}
M_{C R}^{(1)} & -S \\
S^{T} & -M_{C R}^{(0) *}
\end{array}\right)
$$


In the case of fully occupied levels, the diagonal matrices $\bar{O}^{(i)}$ introduced in Eq. (10) tend to infinity and therefore both the determinants $\operatorname{det}\left(\tilde{R}^{(i)}\right)$ and some of the matrix elements of $\tilde{R}^{(i)}$ diverge. The later is not a problem because only the inverse of this matrix is required in Eq. (13) and the inverse

$$
\tilde{R}^{(i)-1}=R^{(i)-1} D^{(i)-1}
$$

is given in terms of $R^{(i)-1}$ which is a well defined quantity in this limit

$$
R^{(i)-1}=\left(\begin{array}{ccc}
\mathbb{I}_{N} & 0 & 0 \\
0 & \mathbb{I}_{N-K^{(i)}} & 0 \\
0 & 0 & 0_{K^{(i)}}
\end{array}\right) .
$$

As a consequence of this structure, the matrix $S$ of Eq. (13) is a matrix where the last $K^{(1)}$ rows and last $K^{(0)}$ columns are zero. This property, together with the special structure of the matrices $M_{C R}^{(i)}$, can be used to reduce the size of the matrices required in the evaluation of the overlap. This simplification is addressed in the next subsection in a slightly different context. The only truly divergent quantities, namely the determinants $\operatorname{det}\left(\tilde{R}^{(i)}\right)$, cancel out in the normalized overlap $\left\langle\phi_{0} \mid \phi_{1}\right\rangle / \sqrt{\left|\left\langle\phi_{0} \mid \phi_{0}\right\rangle\right|\left|\left\langle\phi_{1} \mid \phi_{1}\right\rangle\right|}$ because the norm's modulus $\left|\left\langle\phi_{i} \mid \phi_{i}\right\rangle\right|$ is proportional to $\left|\operatorname{det}\left(\tilde{R}^{(i)}\right)\right|^{2}$.

\section{B. Limit of fully empty levels}

It is often encounter in numerical applications that many orbitals have zero occupancies $v^{2}=0$ and therefore they do not contribute to the overlap. To disentangle those contributions and reduce, in this way, the computational cost of the evaluation of the norm it is convenient to consider the limit of fully empty levels. This limit was considered by other authors [11-14] in the past but in the more traditional formulation of the overlap not including the phase. In the limit of fully empty levels the evaluation of the overlap can be recast in terms of matrices with dimensionality equal to the number of orbitals with nonzero occupancy. This is advantageous as the number of nonzero occupancy levels is usually much smaller than the total dimensionality of the basis used. The same notation as before is used, but now the $\bar{O}^{(i)}$ are diagonal matrices of dimensions $K^{(i)}$ with vanishing diagonal matrix elements. Therefore, the number of empty levels in each HFB wave function $\left|\phi_{i}\right\rangle$ is $2 K^{(i)}$. In this case it is convenient to reorder the single particle basis by using the unitary similarity transformations $P_{23}$ (see Appendix A) permuting blocks 2 and 3, to write $M_{C}^{(i)}=P_{23}^{(i)} M_{C R}^{(i)} P_{23}^{(i)^{T}}$ with

$$
M_{C R}^{(i)}=\left(\begin{array}{cc|cc}
0 & \bar{N}^{(i)} & & \\
-\bar{N}^{(i)} & 0 & & \\
\hline & & 0 & \bar{O}^{(i)} \\
& & -\bar{O}^{(i)} & 0
\end{array}\right)=\left(\begin{array}{cc}
N^{(i)} & 0 \\
0 & O^{(i)}
\end{array}\right),
$$

where the matrices $N^{(i)}$ and $O^{(i)}$ have dimensionality $2(N-$ $\left.K^{(i)}\right) \times 2\left(N-K^{(i)}\right)$ and $2 K^{(i)} \times 2 K^{(i)}$, respectively. The matrices $P_{23}^{(i)}$ carrying out the permutations depend on the HFB wave functions $\left|\phi_{i}\right\rangle$ as the size of each block $\bar{N}^{(i)}$ is not necessarily the same. The impact of this dependence will show up in the evaluation of the pfaffian where the determinant of $P_{23}^{(i)}$ is required. Now, the new unitary matrix $D_{R}^{(i)}=D^{(i)} P_{23}^{(i)}$ is introduced to write

$$
M^{(i)}=D_{R}^{(i)} M_{C R}^{(i)} D_{R}^{(i) T} .
$$

Before proceeding further a technical detail has to be settled down. It is related to the fact that the bipartite structure of $M_{C R}^{(0)}$ differs from the one of $M_{C R}^{(1)}$ as a consequence of the different number of empty levels in each wave function $\left|\phi_{i}\right\rangle$. The simplest case is when both bipartite structures are the same, that is when $K^{(0)}=K^{(1)}$. This motivates the strategy used in the following that consists of assuming the same bipartite structure for each wave function $\left|\phi_{i}\right\rangle$ with a common dimension $K_{S}$ which is the smallest of $K^{(0)}$ and $K^{(1)}$. This strategy implies that some empty levels in one of the wave functions will be treated as if they had a nonzero occupancy. With this in mind, we endow the matrices $M_{C R}^{(i)}$ with the new bipartite structure

$$
M_{C R}^{(i)}=\left(\begin{array}{cc}
N_{C}^{(i)} & 0 \\
0 & O^{(i)}
\end{array}\right),
$$

where $N_{C}^{(i)}$ are $2\left(N-K_{S}\right) \times 2\left(N-K_{S}\right)$ matrices and the $O^{(i)}$ have the same dimension $2 K_{S} \times 2 K_{S}$. In the same way, the same bipartite structure is assumed for the matrix $D_{R}^{(i)}$

$$
D_{R}^{(i)}=\left(\begin{array}{ll}
D_{11}^{(i)} & D_{12}^{(i)} \\
D_{21}^{(i)} & D_{22}^{(i)}
\end{array}\right),
$$

where $D_{11}^{(i)}$ is a square matrix of dimension $2\left(N-K_{S}\right) \times$ $2\left(N-K_{S}\right), D_{12}^{(i)}$ is of dimension $2\left(N-K_{S}\right) \times 2 K_{S}, D_{21}^{(i)}$ is of dimension $2 K_{S} \times 2\left(N-K_{S}\right)$ and finally $D_{22}^{(i)}$ is of dimension $2 K_{S} \times 2 K_{S}$. Using now Eqs. (20) and (21) to reconstruct the matrix $M^{(i)}$ of Eq. (19) it is realized that in the $O^{(i)} \rightarrow 0$ limit the submatrices $D_{12}^{(i)}$ and $D_{22}^{(i)}$ do not enter the final expression of $M^{(i)}$. This freedom is used to choose those "arbitrary" matrices as to simplify some of the results to be obtained below. A possible choice is $D_{12}^{(i)}=0$ and $D_{22}^{(i)}=\mathbb{I}_{2 K_{S}}$ that leads to the matrix denoted $\bar{D}_{R}^{(i)}$ and given by

$$
\bar{D}_{R}^{(i)}=\left(\begin{array}{cc}
D_{11}^{(i)} & 0 \\
D_{21}^{(i)} & \mathbb{I}_{2 K_{S}}
\end{array}\right) .
$$

It has the nice property of having a simple inverse

$$
\bar{D}_{R}^{(i)-1}=\left(\begin{array}{cc}
D_{11}^{(i)-1} & 0 \\
-D_{21}^{(i)} D_{11}^{(i)-1} & \mathbb{I}_{2 K_{S}}
\end{array}\right)
$$

involving only the inverses of the matrices $D_{11}^{(i)}$ which have a moderate dimensionality. This is the property guiding the choice made, as we are implicitly assuming that $N-K_{S} \ll$ $K_{S}$ and the cost of most of the matrix operations grows as the cubic power of its dimensionality. 
With all these definitions, the matrix $\mathbb{M}$ entering the formula for the overlap is written as

$$
\mathbb{M}=\left(\begin{array}{cc}
\bar{D}_{R}^{(1)} & 0 \\
0 & \bar{D}_{R}^{(0) *}
\end{array}\right)\left(\begin{array}{cc}
M_{C R}^{(1)} & -U \\
U^{T} & -M_{C R}^{(0) *}
\end{array}\right)\left(\begin{array}{cc}
\bar{D}_{R}^{(1) T} & 0 \\
0 & \bar{D}_{R}^{(0)+}
\end{array}\right),
$$

where

$$
U=\bar{D}_{R}^{(1)-1}\left(\bar{D}_{R}^{(0)+}\right)^{-1}
$$

(please note that the unitary character of the $D_{R}^{(i)}$ matrices is lost with the introduction of the $\bar{D}_{R}^{(i)}$ ones). Using now the properties of the pfaffian we obtain

$$
\begin{aligned}
\operatorname{pf}(\mathbb{M}) & =\operatorname{det}\left(\bar{D}_{R}^{(1)}\right) \operatorname{det}\left(\bar{D}_{R}^{(0) *}\right) \operatorname{pf}(\tilde{\mathbb{M}}) \\
& =\operatorname{det}\left(D_{11}^{(1)}\right) \operatorname{det}\left(D_{11}^{(0) *}\right) \operatorname{pf}(\tilde{\mathbb{M}})
\end{aligned}
$$

with

$$
\tilde{\mathbb{M}}=\left(\begin{array}{cc}
M_{C R}^{(1)} & -U \\
U^{T} & -M_{C R}^{(0) *}
\end{array}\right) .
$$

Let us now analyze the structure of the block matrix $U$ entering the definition of $\tilde{\mathbb{M}}$. Using Eq. (25) together with Eq. (22) allows to write

$$
U=\left(\begin{array}{ll}
U_{11} & U_{12} \\
U_{21} & U_{22}
\end{array}\right)
$$

with

$$
\begin{aligned}
& U_{11}=\left(D_{R 11}^{(0)+} D_{R 11}^{(1)}\right)^{-1}, \\
& U_{12}=-U_{11} D_{R 21}^{(0)+}, \\
& U_{21}=-D_{R 21}^{(1)} U_{11}, \\
& U_{22}=\mathbb{I}+D_{R 21}^{(1)} U_{11} D_{R 21}^{(0)+} .
\end{aligned}
$$

With this definition, the matrix $\tilde{M}$ of Eq. (51) acquires, in the $O^{(i)} \rightarrow 0$ limit, the block structure

$$
\tilde{\mathbf{M}}=\left(\begin{array}{cc|cc}
N_{C}^{(1)} & 0 & -U_{11} & -U_{12} \\
0 & 0 & -U_{21} & -U_{22} \\
\hline U_{11}^{T} & U_{21}^{T} & -N_{C}^{(0) *} & 0 \\
U_{12}^{T} & U_{22}^{T} & 0 & 0
\end{array}\right) .
$$

This block structure is still not beneficial for the simplification of the corresponding pfaffian and we need to use the exchange matrices defined in Appendix A to exchange blocks 2 and 3

$$
\tilde{\mathbb{M}}_{R}=P_{23} \tilde{\mathbb{M}} P_{23}^{T}=\left(\begin{array}{cc|cc}
N_{C}^{(1)} & -U_{11} & 0 & -U_{12} \\
U_{11}^{T} & -N_{C}^{(0) *} & U_{21}^{T} & 0 \\
\hline 0 & -U_{21} & 0 & -U_{22} \\
U_{12}^{T} & 0 & U_{22}^{T} & 0
\end{array}\right) .
$$

Using the formulas of Appendix B for the pfaffian of a bipartite matrix we obtain

$$
\operatorname{pf}\left(\tilde{\mathbb{M}}_{R}\right)=\operatorname{pf}\left(\begin{array}{cc}
0 & -U_{22} \\
U_{22}^{T} & 0
\end{array}\right)
$$

$$
\times \operatorname{pf}\left[\left(\begin{array}{cc}
N_{C}^{(1)} & -X_{12} \\
X_{12}^{T} & -N_{C}^{(0) *}
\end{array}\right)\right],
$$

where

$$
X_{12}=U_{11}-U_{12} U_{22}^{-1} U_{21} .
$$

The first pfaffian in the right hand side of the above expression is simply given by $(-1)^{K_{S}} \operatorname{det} U_{22}$ whereas the second pfaffian can be computed using again the expression for the pfaffian of a bipartite matrix. Collecting all the terms together we finally obtain

$$
\begin{aligned}
\operatorname{pf}\left(\tilde{\mathbb{M}}_{R}\right)= & (-1)^{K_{S}} \operatorname{det}\left(U_{22}\right) \operatorname{Pf}\left(N_{C}^{(1)}\right) \\
& \times \operatorname{pf}\left(-N_{C}^{(0) *}+X_{12}^{T} N_{C}^{(1)-1} X_{12}\right) .
\end{aligned}
$$

Taking into account that $\operatorname{det}\left(P_{23}\right)=1$ we finally obtain

$$
\begin{aligned}
\operatorname{pf}(\mathbb{M})= & (-1)^{K_{S}} \operatorname{det}\left(D_{11}^{(1)}\right) \operatorname{det}\left(D_{11}^{(0) *}\right) \operatorname{det}\left(U_{22}\right) \operatorname{pf}\left(N_{C}^{(1)}\right) \\
& \times \operatorname{pf}\left(-N_{C}^{(0) *}+X_{12}^{T} N_{C}^{(1)-1} X_{12}\right) .
\end{aligned}
$$

The advantage of this ugly result over the direct evaluation of the pfaffian is the low dimensionality of the $N_{C}^{(1)}, N_{C}^{(0)}$, $D_{11}^{(i)}$ and $U_{11}$ matrices, namely $2\left(N-K_{S}\right)$, which is much smaller than the one of the original problem $(2 N)$. The only large matrix in Eq. (37) is the inverse of $U_{22}$ with dimension $2 K_{S}$, however, this does not pose a challenge as its special structure Eq. (33) is very well adapted to the use of the Sherman-Morrison formulas for the determinant and inverse of this kind of special matrices [28].

The result obtained relies on the existence of the inverses of the matrices $D_{11}^{(1)}$ and $D_{11}^{(0)}$ [i.e., their determinant has to be different from zero, see Eq. (39)]. If this is not the case, a possible strategy is to reduce the number of empty levels (i.e., $K_{S}$ ) increasing in this way the dimensionality of both $D_{11}^{(1)}$ and $D_{11}^{(0)}$ and making more unlikely a linear dependence of the matrix columns. The justification is that in the limit $K_{S}=0$ the $D_{11}^{(i)}$ matrices become unitary and therefore invertible.

\section{Using a different reference vacuum}

From the above discussion, it is clear that the structure and properties of the $M^{(i)}$ matrices is intimately related to the reference vacuum used to express the HFB wave functions $\left|\phi_{i}\right\rangle$. This suggests to change the reference vacuum from the true vacuum, implicitly assumed in the previous discussions, to something else with the hope that the new matrices $\bar{M}^{(i)}$ will acquire a more advantageous structure with little or no fully occupied quasiparticles and a huge number of empty ones comparable to the size of the basis. Matrices with these properties will not require the use of the "fully occupied limit" formulas and will benefit from the reduction in computational burden of the "fully empty limit." As an example, let us consider the case where the $\left|\phi_{i}\right\rangle$ correspond to two HFB wave functions with the same particle number averages and different axial quadrupole deformation parameters $q_{2}^{(i)}$. It seems reasonable to expect that a more appropriate reference vacuum would be the "average" HFB wave function $|\bar{\phi}\rangle$ with the same number of particles mean values and a deformation 
parameter $\bar{q}_{2}$ close to both $q_{2}^{(i)}$ [a reasonable choice could be the mean value $\left.\frac{1}{2}\left(q_{2}^{(0)}+q_{2}^{(1)}\right)\right]$. It is reasonable to expect that, with respect to this reference vacuum $|\bar{\phi}\rangle$, the new $\bar{U}^{(i)}$ amplitudes will be very close to the identity matrix whereas the new $\bar{V}^{(i)}$ amplitudes will be small. In other words, the HFB wave functions $\left|\phi_{i}\right\rangle$ will be represented by a linear combination of quasiparticle excitations of the reference state $|\bar{\phi}\rangle$ weighted with amplitudes that will quickly decrease with the number of quasiparticle excitations involved (i.e., the amplitudes for four quasiparticle excitations will be much smaller than the amplitudes of the two quasiparticle ones, etc.). As a consequence the new $\bar{U}^{(i)}$ and $\bar{V}^{(i)}$ amplitudes will correspond to the "fully empty" limit case discussed in the previous subsection (the calculation will benefit from the computational savings of this limit) and will be away from the problematic "fully occupied" limit of Sec. II A. Considering an alternative reference vacuum can also be beneficial in the extension of these ideas to HFB wave functions with odd number parity.

Let us consider the new reference vacuum $|\bar{\phi}\rangle$ with the associated creation $\bar{\alpha}^{+}$and annihilation $\bar{\alpha}$ quasiparticle operators which are defined in terms of a single-particle basis of creation and annihilation operators by means of linear combinations involving the $\bar{U}$ and $\bar{V}$ amplitudes

$$
\left(\begin{array}{c}
\bar{\alpha} \\
\bar{\alpha}^{+}
\end{array}\right)=\left(\begin{array}{cc}
\bar{U}^{+} & \bar{V}^{+} \\
\bar{V}^{T} & \bar{U}^{T}
\end{array}\right)\left(\begin{array}{c}
a \\
a^{+}
\end{array}\right)=\bar{W}^{+}\left(\begin{array}{c}
a \\
a^{+}
\end{array}\right) .
$$

The same relation holds true for the quasiparticle operators $\alpha^{(i)}$ and $\alpha^{(i)+}$ with amplitudes $W^{(i)}$. Using the unitarity of the matrices $W^{(i)}$ and $\bar{W}$ we can express the set of quasiparticle operators $\alpha^{(i)}$ and $\alpha^{(i)+}$ in terms of the $\bar{\alpha}$ and $\bar{\alpha}^{+}$ones as

$$
\left(\begin{array}{c}
\alpha^{(i)} \\
\alpha^{(i)+}
\end{array}\right)=W^{(i)+} \bar{W}\left(\begin{array}{c}
\bar{\alpha} \\
\bar{\alpha}^{+}
\end{array}\right)=\bar{W}^{(i)+}\left(\begin{array}{c}
\bar{\alpha} \\
\bar{\alpha}^{+}
\end{array}\right) .
$$

The "average" HFB wave function $|\bar{\phi}\rangle$ is characterized by the condition that both $\bar{W}^{(0)}$ and $\bar{W}^{(1)}$ are close to the identity matrix. Using the Thouless theorem we can also express the $\left|\bar{\phi}_{i}\right\rangle$ wave functions (satisfying $\left\langle\bar{\phi} \mid \bar{\phi}_{i}\right\rangle=1$ and therefore differing from the previous $\left|\phi_{i}\right\rangle$ by a normalization factor) in terms of the $|\bar{\phi}\rangle$ reference vacuum

$$
\left|\bar{\phi}_{i}\right\rangle=\exp \left(\frac{1}{2} \sum_{k k^{\prime}} \bar{M}_{k k^{\prime}}^{(i)} \bar{\alpha}_{k}^{+} \bar{\alpha}_{k^{\prime}}^{+}\right)|\bar{\phi}\rangle
$$

with $\bar{M}^{(i)}=\left(\bar{V}^{(i)} \bar{U}^{(i)-1}\right)^{*}$ [not to be confused with the diagonal matrix of Eq. (6)]. These two matrices can be easily computed once the $\bar{W}$ coefficients are given and it is even possible to give an analytical expression [29] in terms of $\bar{M}$ and $M^{(i)}$

$$
\bar{M}^{(i)}=Q\left(\bar{M}^{T}+M^{(i)}\right)\left(\mathbb{I}+\bar{M}^{+} M^{(i)}\right)^{-1}\left(Q^{+}\right)^{-1}
$$

with $Q=\left(\mathbb{I}+\bar{M} \bar{M}^{+}\right)^{-1 / 2}$. All the formulas given above (and below) are equally valid for the wave functions given in the form of Eq. (42) with the amplitudes of Eq. (43).

\section{DIFFERENT SINGLE-PARTICLE BASES}

It is common to deal with HFB wave functions $\left|\phi_{i}\right\rangle$ which are defined in terms of different single-particle basis. The corresponding creation and annihilation operators $a_{k}^{+}(i)$ and $a_{k}(i)$ carry indexes $(i)$ to indicate the HFB wave function they belong to. Those bases are usually not complete and therefore they span different subspaces of the full Hilbert space. As a consequence, the formulas obtained above cannot be used because they rely implicitly on a common basis for the two HFB wave functions [11]. The strategy to overcome this problem is to use a bigger subspace encompassing the two subspaces in order to find an orthogonal basis that can be used to characterize the two HFB wave functions $\left|\phi_{i}\right\rangle$ at the same time. In an early consideration of this problem [11] the whole Hilbert space was used for the bigger subspace. Another possibility, explored in this paper, is to consider the subspace obtained by the union of the two subspaces. In this case, special care has to be taken with the resulting basis, union of the two original bases, as it can be redundant (i.e., it can contain linearly dependent vectors).

The two bases characterizing the HFB wave functions $\left|\phi_{i}\right\rangle$, namely $\left\{a_{k}^{+}(0), k=1, \ldots, N_{(0)}\right\}$ and $\left\{a_{k}^{+}(1), k=\right.$ $\left.1, \ldots, N_{(1)}\right\}$, are defined in terms of single particle creation operators (typically those of the harmonic oscillator basis) and have $N_{(0)}$ and $N_{(1)}$ elements, respectively. It is convenient to introduce of the set of creation operators

$$
A_{\mu}^{+}= \begin{cases}a_{k}^{+}(0) & \mu=k k=1, \ldots, N_{(0)} \\ a_{l}^{+}(1) & \mu=l+N_{(0)} l=1, \ldots, N_{(1)}\end{cases}
$$

embracing the two sets of creation operators of the bases. They satisfy the commutation relations $\left\{A_{\mu}, A_{\nu}^{+}\right\}=\mathcal{N}_{\mu \nu}$ and $\left\{A_{\mu}, A_{v}\right\}=\left\{A_{\mu}^{+}, A_{\nu}^{+}\right\}=0$ where the overlap matrix $\mathcal{N}$ of dimension $\left(N_{(0)}+N_{(1)}\right) \times\left(N_{(0)}+N_{(1)}\right)$ is given in terms of the rectangular matrix $T_{k k^{\prime}}=\left\{a_{k}(0), a_{k^{\prime}}^{+}(1)\right\}=\left\langle-\left|a_{k}(0) a_{k^{\prime}}^{+}(1)\right|-\right\rangle$ by the expression

$$
\mathcal{N}=\left(\begin{array}{cc}
\mathbb{I}_{(0)} & T \\
T^{+} & \mathbb{I}_{(1)}
\end{array}\right) .
$$

The overlap matrix is hermitian, semipositive definite and therefore can be diagonalized by a unitary transformation $D$, i.e.,

$$
\mathcal{N}=\operatorname{Dn} D^{+},
$$

where the diagonal matrix $n$ of the eigenvalues is of dimension $\left(N_{(0)}+N_{(1)}\right) \times\left(N_{(0)}+N_{(1)}\right)$. In order to deal with the zero (or smaller than a given threshold) eigenvalues case (which signals the appearance of linearly dependent basis states) it is convenient to introduce the notation

$$
n=\left(\begin{array}{cc}
\bar{n} & 0 \\
0 & \epsilon
\end{array}\right),
$$

where $\bar{n}$ is the diagonal matrix containing the eigenvalues different from zero (above a given numerical threshold) and $\epsilon$ is the diagonal matrix of dimension $N_{\epsilon}$ containing those eigenvalues with value zero (or smaller than a numerical threshold). It is convenient in the ensuing developments to consider the matrix $\epsilon$ as different from zero and therefore invertible. At the end of the calculations $\epsilon$ will be made to tend 
to zero to obtain the final result. Taking this regularization scheme into account, we can define the square root of the overlap matrix $\mathcal{N}^{1 / 2}=D n^{1 / 2} D^{+}$and its inverse $\mathcal{N}^{-1 / 2}=$ $D n^{-1 / 2} D^{+}$that are required to define the operators

$$
B_{\mu}=\sum_{\nu} \mathcal{N}_{\mu \nu}^{-1 / 2} A_{\nu}
$$

as well as the inverse relation $A_{\mu}=\sum_{\nu} \mathcal{N}_{\mu \nu}^{1 / 2} B_{\nu}$. The creation and annihilation operators $B_{\mu}^{+}$and $B_{\nu}$ satisfy canonical commutation relations $\left\{B_{\mu}, B_{\nu}^{+}\right\}=\left(\mathcal{N}^{-1 / 2} \mathcal{N N}^{-1 / 2}\right)_{\mu \nu}=$ $\delta_{\mu \nu}$. They are introduced to express the HFB wave functions of Eq. (42) in the standard way as

$$
\left|\phi_{i}\right\rangle=\exp \left\{\sum_{\mu \mu^{\prime}} \frac{1}{2} \tilde{N}_{\mu \mu^{\prime}}^{(i)} B_{\mu}^{+} B_{\mu^{\prime}}^{+}\right\}|0\rangle
$$

with the matrices of dimension $\left(N_{(0)}+N_{(1)}\right) \times\left(N_{(0)}+N_{(1)}\right)$

$$
\tilde{N}^{(i)}=\mathcal{N}^{1 / 2+} \tilde{M}_{E}^{(i)} \mathcal{N}^{1 / 2 *}
$$

given in terms of the extended matrices [also of dimension $\left.\left(N_{(0)}+N_{(1)}\right) \times\left(N_{(0)}+N_{(1)}\right)\right]$

$$
\tilde{M}_{E}^{(0)}=\left(\begin{array}{cc}
M^{(0)} & 0 \\
0 & 0
\end{array}\right), \quad \tilde{M}_{E}^{(1)}=\left(\begin{array}{cc}
0 & 0 \\
0 & M^{(1)}
\end{array}\right)
$$

defined in the union subspace (the matrices $M^{(i)}$ are $N_{(i)} \times$ $\left.N_{(i)}\right)$. As the operators $B_{\mu}^{+}$and $B_{v}$ satisfy canonical commutation relations, it is now possible to apply the standard formalism already developed in Ref. [1] [see Eq. (3)] to write

$$
\left\langle\phi_{0} \mid \phi_{1}\right\rangle=S_{N_{(0)}+N_{(1)}} \operatorname{pf}(\tilde{\mathbb{M}}),
$$

where the matrix $\tilde{\mathbb{M}}$ entering the argument of the pfaffian is a $2\left(N_{(0)}+N_{(1)}\right) \times 2\left(N_{(0)}+N_{(1)}\right)$ matrix given in terms of the matrices defined in Eq. (50) as

$$
\tilde{\mathbb{M}}=\left(\begin{array}{cc}
\tilde{N}^{(1)} & -\mathbb{I} \\
\mathbb{I} & -\tilde{N}^{(0) *}
\end{array}\right) .
$$

Using the results of Appendix C (to simplify the notation in the following we consider the size of the two basis to be equal $N_{(0)}=N_{(1)}=N$ ) the norm matrix $\mathcal{N}$ is written as

$$
\mathcal{N}=D\left(\begin{array}{cc}
n_{+} & 0 \\
0 & n_{-}
\end{array}\right) D^{+}
$$

where both the diagonal matrices $n_{ \pm}=\mathbb{I} \pm \bar{\Delta}$ and the unitary transformation $D$ are given in terms of the singular value decomposition (SVD) of the overlap matrix $T=E^{+} \bar{\Delta} F$. Using this definition in Eq. (50) we have

$$
\begin{aligned}
\tilde{N}^{(i)} & =D\left(\begin{array}{cc}
n_{+}^{1 / 2} & 0 \\
0 & n_{-}^{1 / 2}
\end{array}\right) D^{+} \tilde{M}_{E}^{(i)} D^{*}\left(\begin{array}{cc}
n_{+}^{1 / 2} & 0 \\
0 & n_{-}^{1 / 2}
\end{array}\right) D^{T} \\
& =D \tilde{N}_{D}^{(i)} D^{T}
\end{aligned}
$$

which defines the matrices $\tilde{N}_{D}^{(i)}$ as

$$
\tilde{N}_{D}^{(i)}=\left(\begin{array}{cc}
n_{+}^{1 / 2} & 0 \\
0 & n_{-}^{1 / 2}
\end{array}\right) D^{+} \tilde{M}_{E}^{(i)} D^{*}\left(\begin{array}{cc}
n_{+}^{1 / 2} & 0 \\
0 & n_{-}^{1 / 2}
\end{array}\right) .
$$

Please note that these matrices are well defined when some of the eigenvalues (of the $n_{-}$kind) of the norm overlap matrix $\mathcal{N}$ go to zero (this happens when some values of the positive diagonal matrix $\bar{\Delta}$ equal one). Using the explicit form of the matrix $D$ given in Appendix $C$ in terms of the matrices $E$ and $F$ entering the SVD of the matrix $T$ and defining the auxiliary matrices $E_{ \pm}=n_{ \pm}^{1 / 2} E$ and $F_{ \pm}=n_{ \pm}^{1 / 2} F$ we get

$$
\tilde{N}_{D}^{(0)}=\frac{1}{2}\left(\begin{array}{cc}
E_{+} & 0 \\
0 & E_{-}
\end{array}\right)\left(\begin{array}{cc}
M^{(0)} & -M^{(0)} \\
-M^{(0)} & M^{(0)}
\end{array}\right)\left(\begin{array}{cc}
E_{+}^{T} & 0 \\
0 & E_{-}^{T}
\end{array}\right)
$$

and

$$
\tilde{N}_{D}^{(1)}=\frac{1}{2}\left(\begin{array}{cc}
F_{+} & 0 \\
0 & F_{-}
\end{array}\right)\left(\begin{array}{ll}
M^{(1)} & M^{(1)} \\
M^{(1)} & M^{(1)}
\end{array}\right)\left(\begin{array}{cc}
F_{+}^{T} & 0 \\
0 & F_{-}^{T}
\end{array}\right) .
$$

Finally, using known properties of the pfaffian we arrive to the final expression of the overlap in terms of the $\tilde{N}_{D}^{(i)}$

$$
\left\langle\phi_{0} \mid \phi_{1}\right\rangle=(-1)^{N} \operatorname{pf}\left(\begin{array}{cc}
\tilde{N}_{D}^{(1)} & -\mathbb{I} \\
\mathbb{I} & -\tilde{N}_{D}^{(0) *}
\end{array}\right) .
$$

It is convenient to consider the limit where the two bases are connected by means of an unitary transformation (see Appendix C). In this case, the SVD of $T$ is trivial and we have $n_{+}^{1 / 2}=\sqrt{2} \mathbb{I}, n_{-}^{1 / 2}=0, E=\mathbb{I}$ and $F=T$. Using these values we have

$$
\tilde{N}_{D}^{(1)}=\left(\begin{array}{cc}
T M^{(1)} T^{T} & 0 \\
0 & 0
\end{array}\right)
$$

and

$$
\tilde{N}_{D}^{(0)}=\left(\begin{array}{cc}
M^{(0)} & 0 \\
0 & 0
\end{array}\right) .
$$

As a consequence of the zero eigenvalues of the overlap matrix, the matrices $\tilde{N}_{D}^{(i)}$ acquire a bipartite structure where only the upper diagonal block is different from zero. In this case, the matrix in the pfaffian of Eq. (59) becomes a block matrix

$$
\left(\begin{array}{cc|cc}
T M^{(1)} T^{T} & 0 & -\mathbb{I} & 0 \\
0 & 0 & 0 & -\mathbb{I} \\
\hline \mathbb{I} & 0 & -M^{(0) *} & 0 \\
0 & \mathbb{I} & 0 & 0
\end{array}\right) .
$$

The pfaffian of this matrix can be evaluated by using the results of Appendix A. Exchanging blocks 2 and 3 of the matrix brings it to block diagonal form. Once in block diagonal form, the pfaffian can be reduced to the product of the pfaffian of each of the diagonal blocks by using a simplified version of the results of Appendix B for the pfaffian of a bipartite matrix. The final results is

$$
\left\langle\phi_{0} \mid \phi_{1}\right\rangle=(-1)^{N(N+1) / 2} \operatorname{pf}\left(\begin{array}{cc}
T M^{(1)} T^{T} & -\mathbb{I} \\
\mathbb{I} & -M^{(0) *}
\end{array}\right)
$$

as expected (see Ref. [1]). 


\section{CONCLUSIONS}

The formula for the evaluation of the norm overlap of two different HFB wave functions is analyzed in two limits. The first limit corresponds to the situation when some of the occupancies of the quasiparticle levels become one and the standard approach leads to indeterminacies that have to be singled out in order to obtain a meaningful answer. The second limit considers the situation where there are fully empty quasiparticle levels. In this case, the overlap formula is well behaved but considering explicitly the limit leads to a (significant) reduction of the computational burden when the number of empty levels is large enough. The common case where each of the two HFB wave functions are expressed in different single particle basis is also addressed and the formalism to compute the overlap in this situation is developed. A common basis, union of the other two is considered, and special attention is paid to redundancies in the enlarged subspace. The formulas given in this paper are a practical complement of the general one given in [1] and should be useful for a practical implementation of the calculation of the overlaps of two different HFB wave functions in the most general case.

\section{ACKNOWLEDGMENTS}

This work was supported by Ministerio de Ciencia e Innovación (Spain) Grant Nos. FPA2009-08958 and FIS200907277, as well as by Consolider-Ingenio 2010 Programs CPAN CSD2007-00042 and MULTIDARK CSD2009-00064.

\section{APPENDIX A: REORDERING OF MATRICES AND ITS IMPACT IN THE PFAFFIAN}

Very often in this paper the peculiarities of the block structure of the skew-symmetric matrices considered has to be exploited in order to simplify the final expression of the pfaffian. In this respect, it is convenient to know how to reorder rows and columns of a matrix as well as the impact of such reordering in the pfaffian. A useful set of matrices is the one of the matrices $E(i, j)$ that exchanges columns $i$ and $j$ of a matrix when multiplied to the right hand side of that matrix, i.e.,

$$
\begin{aligned}
& \left(\begin{array}{ccccccccc}
\ldots & a_{i-1} & a_{i} & a_{i+1} & \ldots & a_{j-1} & a_{j} & a_{j+1} & \ldots \\
\vdots & \vdots & \vdots & \vdots & \vdots & \vdots & \vdots & \vdots & \vdots
\end{array}\right) E(i, j) \\
& =\left(\begin{array}{ccccccccc}
\ldots & a_{i-1} & a_{j} & a_{i+1} & \ldots & a_{j-1} & a_{i} & a_{j+1} & \ldots \\
\vdots & \vdots & \vdots & \vdots & \vdots & \vdots & \vdots & \vdots & \vdots
\end{array}\right) .
\end{aligned}
$$

The matrices $E(i j)$ are characterized by the matrix elements

$$
E(i, j)_{k l}=\delta_{k l}-\delta_{k i} \delta_{l i}-\delta_{k j} \delta_{l j}+\delta_{k j} \delta_{l i}+\delta_{k i} \delta_{l j}
$$

and are unit matrices where the elements $i$ and $j$ of the diagonal are set to zero and the elements $i, j$ and $j, i$ are set to one.

Another useful set of matrices is the one of the $S(i, j)$ matrices such that, when multiplied to the right-hand side of a matrix, move the column $j$ of the matrix to the position of column $i(i<j)$ and then shifts column $i$ to position $i+1$, column $i+1$ to position $i+2$, and so on, up to column $j-1$ that is shifted to column $j$, i.e.,

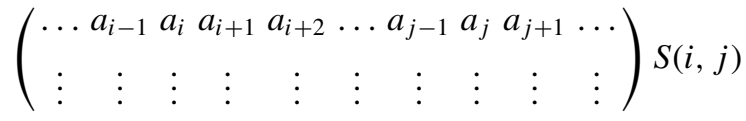

$$
\begin{aligned}
& =\left(\begin{array}{cccccccccc}
\ldots & a_{i-1} & a_{j} & a_{i} & a_{i+1} & a_{i+2} & \ldots & a_{j-1} & a_{j+1} & \ldots \\
\vdots & \vdots & \vdots & \vdots & \vdots & \vdots & \vdots & \vdots & \vdots & \vdots
\end{array}\right) .
\end{aligned}
$$

In terms of matrix elements they are given by

$$
S(i, j)_{k l}=\delta_{k l}-\sum_{s=i}^{j} \delta_{k s} \delta_{l s}+\sum_{s=i}^{j-1} \delta_{k s} \delta_{l s+1}+\delta_{k j} \delta_{l i} .
$$

These matrices are unit matrices where the 1 in position $i, i$ is shifted to position $i, i+1$, the 1 in position $i+1, i+1$ is shifted to position $i+1, i+2$ and so on up the 1 in position $j, j$ that is shifted to position $j, i$. The determinants of the two kind of matrices are easy to determine and they are given by

$$
\operatorname{det}[E(i, j)]=-1
$$

and

$$
\operatorname{det}[S(i, j)]=(-1)^{j-i} .
$$

The successive application of the matrices $E(i+k, j+k)$ for $k=0$ up to $k=N$ defines a matrix

$$
P_{N}(i, j)=\prod_{k=0}^{N-1} E(i+k, j+k)
$$

that exchanges a set of $N$ columns at once

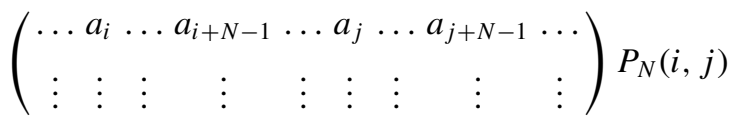

$$
\begin{aligned}
& =\left(\begin{array}{ccccccccc}
\ldots & a_{j} & \ldots & a_{j+N-1} & \ldots & a_{i} & \ldots & a_{i+N-1} & \ldots \\
\vdots & \vdots & \vdots & \vdots & \vdots & \vdots & \vdots & \vdots & \vdots
\end{array}\right) .
\end{aligned}
$$

Applying the matrix $P_{N}^{T}$ to the left of the matrix the corresponding exchange of rows is produced. As a consequence

$$
\begin{aligned}
P_{N}^{T}(i, j) & \left(\begin{array}{ccccc}
\ddots & \vdots & \vdots & \vdots & \\
\cdots & A_{N}(i, i) & \cdots & A_{N}(i, j) & \cdots \\
\vdots & \vdots & \ddots & \vdots & \vdots \\
\cdots & A_{N}(j, i) & \cdots & A_{N}(j, j) & \cdots \\
& \vdots & \vdots & \vdots & \ddots
\end{array}\right) P_{N}(i, j) \\
= & \left(\begin{array}{ccccc}
\ddots & \vdots & \vdots & \vdots & \\
\cdots & A_{N}(j, j) & \cdots & A_{N}(j, i) & \cdots \\
\vdots & \vdots & \ddots & \vdots & \vdots \\
\cdots & A_{N}(i, j) & \cdots & A_{N}(i, i) & \cdots \\
& \vdots & \vdots & \vdots & \ddots
\end{array}\right), \quad(\mathrm{A} 9)
\end{aligned}
$$


where $A_{N}(i, j)$ are submatrices of dimension $N \times N$ whose first element is located in the row $i$ and column $j$ of the matrix where the $A_{N}$ are embedded. The result obtained, together with that of the pfaffian of a bipartite matrix, will be useful to reduce some of the pfaffians encountered in the main body of the paper. Obviously $\operatorname{det} P_{N}=(-1)^{N}$. Unfortunately, this trick cannot be applied when the number of columns to be "exchanged" is not the same. In such case, we have to consider the more general shift operation $S(i, j)$. To see how it works let us consider a matrix $A$ were there are three groups of columns denoted by $L, C$, and $R$ such that the first group goes from column $i$ to columns $i+N_{L}-1$ (i.e., $N_{L}$ columns), the group $C$ goes from column $i+N_{L}$ up to columns $i+N_{L}+N_{C}-$ 1 (i.e., $N_{C}$ columns) and finally the group $R$ from column $i+N_{L}+N_{C}$ up to column $i+N_{L}+N_{C}+N_{R}-1$ (i.e., $N_{R}$ columns). Schematically, the columns of the matrix $A$ could be represented as

$$
A=(\ldots|L| C|R| \ldots)
$$

Now we want to exchange the group $L$ with the group $R$; to do so the group $R$ of columns is moved to the position occupied by $L$ using the product of matrices

$$
P_{L R}^{(1)}=\prod_{k=0}^{N_{R}-1} S\left(i+k, i+k+N_{L}+N_{C}\right)
$$

giving

$$
(\ldots|L| C|R| \ldots) P_{L R}^{(1)}=(\ldots|R| L|C| \ldots) .
$$

Now, the group of columns $C$ is moved to the position of the group $L$ by means of the following product of "shift" matrices

$$
P_{L C}=\prod_{k=0}^{N_{C}-1} S\left(i+k+N_{R}, i+k+N_{R}+N_{L}\right) .
$$

Using this matrix we obtain

$$
\begin{aligned}
& (\ldots|L| C|R| \ldots) P_{L R}^{(1)} P_{L C}=(\ldots|R| L|C| \ldots) P_{L C} \\
& \quad=(\ldots|R| C|L| \ldots) .
\end{aligned}
$$

The matrix exchanging the set of columns $L$ with the set $R$ will be denoted $P_{L R}=P_{L R}^{(1)} P_{L C}$. By applying $P_{L R}^{T}$ to the left of the matrix the set of rows $L$ and $R$ are exchanged. As a consequence

$$
P_{L R}^{T}(i, j)\left(\begin{array}{ccccc}
\ddots & \vdots & \vdots & \vdots \\
\cdots & A_{N_{L} \times N_{L}}(i, i) & \cdots & A_{N_{L} \times N_{R}}(i, j) & \cdots \\
\vdots & \vdots & \ddots & \vdots & \vdots \\
\cdots & A_{N_{R} \times N_{L}}(j, i) & \cdots & A_{N_{R} \times N_{R}}(j, j) & \cdots \\
& \vdots & \vdots & \vdots & \ddots
\end{array}\right)\left(\begin{array}{cccccc}
\ddots & \vdots & \vdots & \\
\cdots & A_{N_{R} \times N_{R}}(j, j) & \cdots & A_{N_{R} \times N_{L}}(j, i) & \cdots \\
\vdots & \vdots & \ddots & \vdots \\
\cdots & A_{N_{L} \times N_{R}}(i, j) & \cdots & A_{N_{L} \times N_{L}}(i, i) & \cdots \\
\vdots & \vdots & \vdots & \ddots
\end{array}\right)
$$

where $A_{N_{L} \times N_{L}}(i, i)$ is a submatrix with $N_{L}$ rows and $N_{L}$ columns starting at row $i$ and column $i, A_{N_{L} \times N_{R}}(i, j)$ is a submatrix with $N_{L}$ rows and $N_{R}$ columns starting at row $i$ and column $j=i+N_{L}+N_{C}$, and so on. This result generalizes the one of Eq. (A9) for submatrices of different sizes. The impact of such exchange of rows and columns in the pfaffian is the product of the determinants of the $S$ matrices involved, i.e., $\operatorname{det}\left(P_{L R}\right)=\operatorname{det}\left(P_{L R}^{(1)}\right) \operatorname{det}\left(P_{L C}\right)$. With $\operatorname{det}\left(P_{L R}^{(1)}\right)=(-1)^{\left(N_{L}+N_{C}\right) N_{R}}$ and $\operatorname{det}\left(P_{L C}\right)=(-1)^{N_{L} N_{C}}$ the total phase is $\operatorname{det}\left(P_{L R}\right)=(-1)^{N_{L} N_{R}+N_{C} N_{R}+N_{L} N_{C}}$ that reduces to $(-1)^{N}$ when $N_{L}=N_{R}=N$, a result that is independent of $N_{C}$.

\section{APPENDIX B: THE PFAFFIAN OF A BIPARTITE MATRIX}

To derive some of the results obtained in this paper, it is often required to compute the pfaffian of a bipartite skewsymmetric matrix with the general structure

$$
S=\left(\begin{array}{cc}
M & Q \\
-Q^{T} & N
\end{array}\right)
$$

where $M$ and $N$ are skew-symmetric matrices and $Q$ is a general rectangular matrix. Using Aitken's formula it is possible to diagonalize the bipartite matrix using a congruence transformation [18]

$$
\begin{aligned}
& \left(\begin{array}{cc}
\mathbb{I} & 0 \\
Q^{T} M^{-1} & \mathbb{I}
\end{array}\right)\left(\begin{array}{cc}
M & Q \\
-Q^{T} & N
\end{array}\right)\left(\begin{array}{cc}
\mathbb{I} & -M^{-1} Q \\
0 & \mathbb{I}
\end{array}\right) \\
& =\left(\begin{array}{cc}
M & 0 \\
0 & N+Q^{T} M^{-1} Q
\end{array}\right) .
\end{aligned}
$$

An equivalent expression is given by

$$
\begin{aligned}
& \left(\begin{array}{cc}
\mathbb{I} & -Q N^{-1} \\
0 & \mathbb{I}
\end{array}\right)\left(\begin{array}{cc}
M & Q \\
-Q^{T} & N
\end{array}\right)\left(\begin{array}{cc}
\mathbb{I} & 0 \\
N^{-1} Q^{T} & \mathbb{I}
\end{array}\right) \\
& \quad=\left(\begin{array}{cc}
M+Q N^{-1} Q^{T} & 0 \\
0 & N
\end{array}\right)
\end{aligned}
$$

that is useful if the matrix $M^{-1}$ does not exist. This block diagonalization formula involves congruence transformations that allow to use the property $\operatorname{pf}\left(P^{T} R P\right)=\operatorname{det}(P) \operatorname{pf}(R)$ of the 
pfaffian to obtain the next two identities

$$
\begin{aligned}
\operatorname{pf}(S) & =\operatorname{pf}(M) \operatorname{pf}\left(N+Q^{T} M^{-1} Q\right) \\
& =\operatorname{pf}\left(M+Q N^{-1} Q^{T}\right) \operatorname{pf}(N) .
\end{aligned}
$$

\section{APPENDIX C: THE OVERLAP MATRIX}

The overlap matrix $\mathcal{N}$ has the bipartite structure

$$
\mathcal{N}=\left(\begin{array}{cc}
\mathbb{I}_{(0)} & T \\
T^{+} & \mathbb{I}_{(1)}
\end{array}\right)
$$

where the rectangular matrix $T$, with matrix elements $T_{k k^{\prime}}=$ $\left\{a_{k}(0), a_{k^{\prime}}^{+}(1)\right\}=\left\langle 0\left|a_{k}(0) a_{k^{\prime}}^{+}(1)\right| 0\right\rangle$, is the matrix of the overlaps between the elements of the two basis considered. As in the body of the paper, $N_{(0)}$ and $N_{(1)}$ denote the dimensions of the each of the basis and it is assumed for definiteness that $N_{(0)} \geqslant N_{(1)}$. The matrices $\mathbb{I}_{(0)}$ and $\mathbb{I}_{(1)}$ stand for the identity matrices of dimensions $N_{(0)}$ and $N_{(1)}$, respectively. For the developments considered in this paper, the analysis of the spectral decomposition of the overlap matrix is required in order to handle properly the occurrence of very small or zero eigenvalues of the overlap. The analysis is based on the singular value decomposition (SVD) [30] of the matrix $T$

$$
T=E^{+} \Delta F,
$$

where $E$ and $F$ are square unitary matrices of dimensions $N_{(0)} \times N_{(0)}$ and $N_{(1)} \times N_{(1)}$, respectively. The matrix $\Delta$ is a rectangular matrix of dimension $N_{(0)} \times N_{(1)}$ with the "diagonal structure"

$$
\Delta=\left(\begin{array}{c}
\bar{\Delta} \\
0
\end{array}\right),
$$

where $\bar{\Delta}$ is a real and positive square diagonal matrix with dimension $N_{(1)} \times N_{(1)}$. It is convenient to introduce a rectangular "identity matrix" $\mathbb{I}_{(01)}$ of dimension $N_{(0)} \times N_{(1)}$ with a structure similar to the one of the matrix $\Delta$, namely,

$$
\mathbb{I}_{(01)}=\left(\begin{array}{c}
\mathbb{I}_{(1)} \\
0
\end{array}\right) \text {. }
$$

Useful properties of this matrix are $\Delta=\mathbb{I}_{(01)} \bar{\Delta}$ and $\mathbb{I}_{(01)}^{+} \mathbb{I}_{(01)}=$ $\mathbb{I}_{(1)}$.

Using the SVD of $T$ defined in Eq. (C2) we can finally write

$$
\mathcal{N}=\bar{D}\left(\begin{array}{cc}
\mathbb{I}_{(0)} & \Delta \\
\Delta^{+} & \mathbb{I}_{(1)}
\end{array}\right) \bar{D}^{+}
$$

where

$$
\bar{D}=\left(\begin{array}{cc}
E^{+} & 0 \\
0 & F^{+}
\end{array}\right)
$$

The matrix in the middle of the right-hand side of Eq. (C5) can be easily brought to diagonal form

$$
\begin{aligned}
\bar{D}^{(0)+}\left(\begin{array}{cc}
\mathbb{I}_{(0)} & \Delta \\
\Delta^{+} & \mathbb{I}_{(1)}
\end{array}\right) \bar{D}^{(0)} \\
=\left(\begin{array}{cc}
2 \mathbb{I}_{(0)}+\mathbb{I}_{(01)}\left(\bar{\Delta}-\mathbb{I}_{(1)}\right) \mathbb{I}_{(01)}^{+} & 0 \\
0 & \mathbb{I}_{(1)}-\bar{\Delta}
\end{array}\right)
\end{aligned}
$$

by means of the $\bar{D}^{(0)}$ transformation

$$
\bar{D}^{(0)}=\frac{1}{\sqrt{2}}\left(\begin{array}{cc}
\mathbb{I}_{(0)} & -\mathbb{I}_{(01)} \\
\mathbb{I}_{(01)}^{+} & \mathbb{I}_{(1)}
\end{array}\right) .
$$

Finally, introducing the matrix $D=\bar{D} \bar{D}^{(0)}$ we obtain the complete diagonalization of the overlap matrix

$$
\mathcal{N}=D\left(\begin{array}{cc}
2 \mathbb{I}_{(0)}+\mathbb{I}_{(01)}\left(\bar{\Delta}-\mathbb{I}_{(1)}\right) \mathbb{I}_{(01)}^{+} & 0 \\
0 & \mathbb{I}_{(1)}-\bar{\Delta}
\end{array}\right) D^{+} .
$$

The semipositive character of the matrix $\mathcal{N}$ implies that all its eigenvalues are positive or zero and therefore (lower block) the elements of the diagonal matrix $\bar{\Delta}$ cannot exceed one. In the case of bases with equal dimensions $N_{(0)}=N_{(1)}$ the above result becomes

$$
\mathcal{N}=D\left(\begin{array}{cc}
\mathbb{I}+\bar{\Delta} & 0 \\
0 & \mathbb{I}-\bar{\Delta}
\end{array}\right) D^{+}=D\left(\begin{array}{cc}
n_{+} & 0 \\
0 & n_{-}
\end{array}\right) D^{+},
$$

where the diagonal matrices $n_{ \pm}=\mathbb{I} \pm \bar{\Delta}$ are introduced. As the matrix $\bar{\Delta}$ is also positive semidefinite it is clear that the zero norm eigenvalues of $\mathcal{N}$ are associated to the matrix elements of $\bar{\Delta}$ equal to one.

It is helpful to consider the special case where $T$ is an unitary matrix. It corresponds to the common situation where the two bases are connected through a unitary transformation as, for instance, the ones associated to symmetry operations like rotations in real space, etc., acting on closed basis. For unitary $T$, the SVD factors can be chosen as $E^{+}=T, \Delta=\mathbb{I}$ and $F=\mathbb{I}$ in the formulas above so that

$$
D=\frac{1}{\sqrt{2}}\left(\begin{array}{cc}
T & -T \\
\mathbb{I} & \mathbb{I}
\end{array}\right)
$$

and

$$
\mathcal{N}=D\left(\begin{array}{cc}
2 \mathbb{I} & 0 \\
0 & 0
\end{array}\right) D^{+}
$$

with $N$-fold degenerate eigenvalues $n_{+}=2$ and $n_{-}=0$.

To finish this appendix, we just mention that a similar (but less general) treatment of the norm overlap was considered in Ref. [31] but without resorting to the powerful concept of the SVD.
[1] L. M. Robledo, Phys. Rev. C 79, 021302(R) (2009).

[2] N. Onishi and S. Yoshida, Nucl. Phys. 80, 367 (1966).

[3] K. Neergard and E. Wüst, Nucl. Phys. A 402, 311 (1983).
[4] John R. Klauder and Bo-Sture Skagerstam, Coherent States: Applications in Physics and Mathematical Physics (World Scientific, Singapore, 1985). 
[5] F. A. Berezin, The Method of Second Quantization (Academic Press, New York, 1966).

[6] J.-P. Blaizot and G. Ripka, Quantum Theory of Finite Systems (MIT Press, Cambridge, MA/London, 1985).

[7] J. W. Negele and H. Orland, Quantum Many Particle Systems (Addison-Wesley, Redwood City, CA, 1988).

[8] Y. Ohnuki and T. Kashiwa, Prog. Theor. Phys. 60, 548 (1978).

[9] E. R. Caianiello, Combinatorics and Renormalization in Quantum Field Theory (W. A. Benjamin, Reading, MA, 1973).

[10] P. Ring and P. Schuck, The Nuclear Many Body problem (Springer-Verlag, Berlin, 1980).

[11] L. M. Robledo, Phys. Rev. C 50, 2874 (1994).

[12] J. M. Yao, J. Meng, P. Ring, and D. Pena Arteaga, Phys. Rev. C 79, 044312 (2009).

[13] P. Bonche, J. Dobaczewski, H. Flocard, P.-H. Heenen, and J. Meyer, Nucl. Phys. A 510, 466 (1990).

[14] Q. Haider and D. Gogny, J. Phys. G 18, 993 (1992).

[15] M. Bajdich, L. Mitas, and L. K. Wagner, and K. E. Schmidt, Phys. Rev. B 77, 115112 (2008).

[16] C. K. Thomas and A. A. Middleton, Phys. Rev. E 80, 046708 (2009).

[17] J.-M. Stéphan, S. Furukawa, G. Misguich, and V. Pasquier, Phys. Rev. B 80, 184421 (2009).
[18] C. Gonzalez-Ballestero, L. M. Robledo, and G. F. Bertsch, arXiv:1012.5022 (submitted to Comp. Phys. Comm.).

[19] M. Bender, P.-H. Heenen, and P.-G. Reinhard, Rev. Mod. Phys. 75, 121 (2003).

[20] R. R. Rodríguez-Guzmán, J. L. Egido, and L. M. Robledo, Nucl. Phys. A 709, 201 (2002).

[21] S. Perez-Martin and L. M. Robledo, Phys. Rev. C 76, 064314 (2007).

[22] K. W. Schmid, Prog. Part. Nucl. Phys. 52, 565 (2004).

[23] M. Bender and P.-H. Heenen, Phys. Rev. C 78, 024309 (2008).

[24] M. Oi and N. Tajima, Phys. Lett. B 606, 43 (2005).

[25] F. Dönau, Phys. Rev. C 58, 872 (1998).

[26] M. Anguiano, J. L. Egido, and L. M. Robledo, Nucl. Phys. A 696, 467 (2001).

[27] D. Lacroix, T. Duguet, and M. Bender, Phys. Rev. C 79, 044318 (2009).

[28] William H. Press, Saul A. Teukolsky, William T. Vetterling, and Brian P. Flannery, Numerical Recipes: The Art of Scientific Computing, 3rd ed. (Cambridge University Press, Cambridge, 2007).

[29] J. P. Blaizot and H. Orland, Phys. Rev. C 24, 1740 (1981).

[30] G. H. Golub and C. F. Van Loan, Matrix Computations (Johns Hopkins University Press, Baltimore, 1996).

[31] J. F. Berger and D. Gogny, Nucl. Phys. A 333, 302 (1980). 